ORIGINAL ARTICLE

\title{
Estabilidade química, físico-química e antioxidante de polpa de Physalis pasteurizada e não pasteurizada sob congelamento
}

\section{Chemical, physicochemical and antioxidant stability of freezing pasteurized and unpasteurized pulp}

\author{
Taiana Ferreira Machado ${ }^{1}$, Eduarda Rodrigues Monteiro ${ }^{1}$, Aline Tiecher $^{1 *}$ (i) \\ ${ }^{1}$ Universidade Federal do Pampa, Ciência e Tecnologia de Alimentos (UNIPAMPA), Itaqui/RS - Brasil
}

*Corresponding Author: Aline Tiecher, Universidade Federal do Pampa, Ciência e Tecnologia de Alimentos (UNIPAMPA), R. Tito Corrêa Lopes, s/n, Promorar, CEP: 97650-000, Itaqui/RS - Brasil, e-mail:

atiecher.alimentos@gmail.com

Cite as: Machado, T. F., Monteiro, E. R., \& Tiecher, T. (2019). Chemical, physicochemical and antioxidant stability of freezing pasteurized and unpasteurized pulp. Brazilian Journal of Food Technology, 22, e2017149.

https://doi.org/10.1590/1981-6723.14917

\begin{abstract}
Resumo
O trabalho teve como objetivo avaliar a estabilidade físico-química, química e antioxidante de polpa de Physalis peruviana L., não pasteurizada e pasteurizada, durante 120 dias de armazenamento a $-18{ }^{\circ} \mathrm{C}$. Foram avaliados $\mathrm{pH}$, acidez total titulável (ATT), sólidos solúveis totais (SST), relação SST/ATT, umidade, cinzas, glicídeos redutores, coloração, vitamina C, carotenoides totais, compostos fenólicos totais e capacidade antioxidante. As análises físicoquímicas e químicas realizadas demonstraram que o armazenamento sob congelamento não ocasionou perdas significativas de qualidade das polpas para as variáveis de $\mathrm{pH}$, ATT, SST e glicídeos redutores. Na polpa não pasteurizada, observou-se aumento significativo no teor de umidade e diminuição significativa no teor de cinzas, durante o armazenamento. A aplicação da pasteurização promoveu variações significativas para os SST e glicídios redutores, ao final do período de armazenamento, observando-se maiores teores. A cor foi afetada negativamente pela aplicação do tratamento térmico, observando-se valores de $\mathrm{a}^{*}$ inferiores e valores do ângulo da tonalidade $\left({ }^{\circ} \mathrm{h}\right)$ superiores. Quanto à estabilidade dos compostos antioxidantes, verificou-se redução nos teores de vitamina $\mathrm{C}$, carotenoides, compostos fenólicos totais e da capacidade antioxidante, ao longo do armazenamento a $-18{ }^{\circ} \mathrm{C}$, nas polpas não pasteurizada e pasteurizada. Foram observadas perdas de $82,38 \%$ e $88,88 \%$ para vitamina C, de $66,29 \%$ e $66,51 \%$ para os carotenoides, e 22,26\% e 31,31\% para os compostos fenólicos totais nas polpas não pasteurizadas e pasteurizadas, respectivamente. Conclui-se que o armazenamento durante 120 dias sob congelamento das polpas de Physalis peruviana L. pasteurizada e não pasteurizada promoveu perdas significativas dos compostos antioxidantes para ambas as polpas e que a pasteurização afetou as características físico-químicas e químicas avaliadas.
\end{abstract}

Palavras-chave: Physalis peruviana L.; Pasteurização; Coloração; Carotenoides; Capacidade antioxidante; Sólidos solúveis totais. 


\begin{abstract}
The aim of this work was to evaluate the physicochemical, chemical and antioxidant stability of unpasteurized and pasteurized Physalis peruviana L. pulp during 120 days of storage at $-18^{\circ} \mathrm{C}$. Total titratable acidity (TTA), total soluble solids (TSS), TSS/TTA ratio, $\mathrm{pH}$, moisture, ash, reducing sugars, color, vitamin $\mathrm{C}$, total carotenoids, total phenolic compounds and antioxidant capacity were evaluated. The physicochemical and chemical analysis showed that storage under freezing did not cause significant loss of pulp quality for $\mathrm{pH}$, TTA, TSS and reducing sugars. In the unpasteurized pulp, there was a significant increase in moisture content and a significant decrease in the ash content along the days of storage. The application of pasteurization promoted significant variations for the TSS and reducing sugars, at the end of the storage period, observing higher levels. The color was negatively affected by the heat treatment, observing lower values of $a^{*}$ and higher values of hue angle $\left({ }^{\circ} \mathrm{h}\right)$. As regards the stability of antioxidant compounds, there was a reduction in vitamin $\mathrm{C}$, carotenoids, total phenolic compounds and antioxidant capacity during storage at $-18{ }^{\circ} \mathrm{C}$ in unpasteurized and pasteurized pulps. There were losses of $82.38 \%$ and $88.88 \%$ for vitamin C, $66.29 \%$ and $66.51 \%$ for carotenoids, and $22.26 \%$ and $31.31 \%$ for total phenolic compounds in unpasteurized and pasteurized pulps, respectively. It was concluded that the storage under freezing of pasteurized and unpasteurized Physalis peruviana L. pulp and pasteurization affected the physicochemical and chemical characteristics evaluated.
\end{abstract}

Keywords: Physalis peruviana L.; Pasteurization; Color; Carotenoids; Antioxidant capacity; Total soluble solids.

\title{
1 Introdução
}

Physalis peruviana L. é um semiarbusto pertencente à família Solanaceae, originária do Peru (Fischer et al., 2011), possuindo variedades cultivadas nos continentes América, Europa e Ásia (Rockenbach et al., 2008). O fruto é uma baga carnosa, com peso aproximado de 4 a $5 \mathrm{~g}$, que apresenta forma esférica e se desenvolve no interior de um cálice, o qual possui a finalidade de preservá-lo (Ramadan, 2011). Após a remoção do cálice, o fruto apresenta um período de armazenamento reduzido. Bolzan et al. (2011), ao avaliarem o armazenamento refrigerado de physalis, verificaram que os melhores resultados são obtidos quando os frutos são armazenados com cálice.

Os frutos de physalis têm sido considerados uma boa fonte de provitamina A, minerais, vitamina $\mathrm{C}$ e do complexo B (Ramadan, 2011). Cada $100 \mathrm{~g}$ de physalis apresenta cerca de 78,9 g de umidade, 0,05 a 0,3 g de proteína, 0,15 a 0,2 g de lipídeos, 19,6 g de carboidratos, 4,9 g de fibras e $1 \mathrm{~g}$ de cinzas (Ramadan \& Mörsel, 2004). Silva et al. (2013) encontraram um teor de ácido ascórbico de 25,55 mg por 100 g. Com relação ao $\mathrm{pH}$, acidez total titulável e sólidos solúveis totais, Rodrigues et al. (2014) encontraram, respectivamente, $3,46 \pm 0,14,1,57 \% \pm 0,03 \%$ de ácido cítrico e $13,81 \pm 0,05^{\circ}$ Brix.

Além da importância nutricional, o fruto de physalis contém compostos não nutrientes, denominados compostos antioxidantes, que apresentam efeitos fisiológicos e/ou metabólicos no organismo, tais como os carotenoides e compostos fenólicos. Estudos realizados por Rockenbach et al. (2008) encontraram teores de compostos fenólicos totais obtidos para extrato metanólico de 57,9 mg de equivalentes a ácido gálico (GAE) por $100 \mathrm{~g} \mathrm{em}$ fruto de physalis e Oliveira (2016) encontrou 5,95 $\mu \mathrm{g}$ por g de carotenoides nos frutos in natura.

No Brasil, a comercialização de physalis é realizada a preços altos, visto que a produção comercial no país é pequena. Na maioria dos casos, a fruta é cultivada em quintais para o consumo direto (Rockenbach et al., 2008). A colheita dos frutos se inicia entre três e cinco meses após o transplante das mudas e, uma vez iniciada, a colheita deve ser contínua e semanal (Lima et al., 2013). Por se tratar de um fruto climatérico (Fischer et al., 2011), com elevada produção durante o período de colheita, buscam-se alternativas tecnológicas para aumentar a vida de prateleira dos frutos, sendo o processamento de polpa uma forma de aproveitamento. A polpa produzida poderá ser utilizada como ingrediente para obtenção de outros produtos, sem que seja necessário realizar etapas de processamento adicionais (Carvalho et al., 2017).

Segundo a Instrução Normativa n. ${ }^{\circ}$ 01, de 07 de janeiro de 2000, do Ministério da Agricultura Pecuária e Abastecimento (MAPA), polpa de fruta é o produto não fermentado, não concentrado, não diluído, obtido de 
frutos polposos, através de processo tecnológico adequado, com um teor mínimo de sólidos totais, proveniente da parte comestível do fruto (Brasil, 2000).

O processamento de polpas de frutas segue algumas etapas básicas, como recepção, seleção, lavagem, sanitização, despolpamento e armazenamento (Matta et al., 2005). Para a conservação, as polpas de frutas são submetidas a diversos métodos, entre os quais se destacam o processamento por remoção do calor (congelamento) e por aplicação de calor (pasteurização) (Fellows, 2006). Esses métodos aumentam a vida de prateleira. No entanto, acontecem pequenas mudanças nas características sensoriais, nos valores nutricional e funcional, conduzindo a uma redução no nível de compostos antioxidantes (Silva et al., 2015; Rawson et al., 2011).

Diante disso, este trabalho teve como objetivo avaliar a estabilidade química, físico-química e antioxidante de polpa de Physalis peruviana L. pasteurizada e não pasteurizada, armazenadas sob congelamento $\left(-18^{\circ} \mathrm{C}\right)$ durante 120 dias.

\section{Material e métodos}

\subsection{Obtenção da polpa de Physalis}

Frutos de physalis foram adquiridos na Central de Abastecimento do Rio Grande do Sul (CEASA), na cidade de Porto Alegre, e transportados em caixas térmicas até a Universidade Federal do Pampa, campus Itaqui, Rio Grande do Sul. Os frutos foram retirados do cálice, selecionados de acordo com o estádio de maturação, ausência de danos mecânicos e podridões, lavados em água corrente e sanitizados em solução de hipoclorito de sódio a 200 ppm durante 15 minutos. A polpa foi obtida através da trituração dos frutos inteiros em liquidificador. Essa polpa foi fracionada em duas partes. Uma das partes passou pelo processo de pasteurização, que se fundamentou no aquecimento da polpa a $95{ }^{\circ} \mathrm{C}$ durante 15 segundos (Lima et al., 2012). Em seguida, as polpas foram acondicionadas em potes de polipropileno com tampa, com três repetições para cada tempo de amostragem $(0,30,60,90$ e 120 dias $)$, e mantidas sob congelamento a $-18^{\circ} \mathrm{C}$.

\subsection{Análises físico-químicas e químicas}

Todas as análises foram realizadas em triplicata e realizadas de acordo com a metodologia prescrita pelo Instituto Adolfo Lutz (2008).

\subsubsection{Determinação de pH, Acidez Total Titulável (ATT) e de Sólidos Solúveis Totais (SST)}

$\mathrm{O} \mathrm{pH}$ foi determinado com uso de um pHmetro digital. A ATT foi determinada por volumetria potenciométrica, com resultados expressos em porcentagem (\%) de ácido cítrico. Os SST foram determinados por refratometria, com resultados expressos em ${ }^{\circ}$ Brix.

\subsubsection{Determinação dos parâmetros instrumentais de cor}

A coloração foi medida com o emprego de colorímetro no padrão CIE- L*a*b*. L* expressa os valores de luminosidade, $\mathrm{a}^{*}$ representa as cores vermelha $(+)$ ou verde $(-)$ e $b^{*}$, as cores amarela $(+)$ ou azul (-). Para calcular o ângulo da tonalidade $\left({ }^{\circ} \mathrm{h}\right)$, que define a tonalidade de cor, foram utilizados os valores de $\mathrm{a}^{*} \mathrm{e} \mathrm{b}^{*}$ $\left[{ }^{\circ} \mathrm{h}=\tan -1(\mathrm{~b} * / \mathrm{a} *)\right]$. 


\subsubsection{Determinação de cinzas e de umidade}

As cinzas foram obtidas através da incineração da amostra em mufla a $550^{\circ} \mathrm{C}$ até peso constante. A perda por dessecação (umidade) foi realizada através de secagem direta em estufa a $105{ }^{\circ} \mathrm{C}$ até peso constante, sendo ambos os resultados expressos em porcentagem $(\%)$.

\subsubsection{Determinação de glicídios redutores}

A determinação de açúcares redutores em glicose foi realizada pelo método volumétrico, mediante titulação com solução de Fehling, expressos em porcentagem (\%) de glicose.

\subsubsection{Determinação de vitamina C}

A determinação de vitamina $\mathrm{C}$ foi realizada através de titulação com iodato de potássio $0,002 \mathrm{M}$, sendo os resultados expressos em miligramas de vitamina $\mathrm{C}$ por 100 gramas de amostra $\left(\mathrm{mg} 100 \mathrm{~g}^{-1}\right)$.

\subsection{Determinação de carotenoides totais, compostos fenólicos totais e capacidade antioxidante}

O método de extração de carotenoides totais seguiu os procedimentos descritos por Rodriguez-Amaya (2001). A quantificação dos carotenoides totais foi determinada a $450 \mathrm{~nm}$ em espectrofotômetro e os resultados expressos em miligramas de $\beta$-caroteno por 100 gramas de amostra fresca $\left(\mathrm{mg} 100 \mathrm{~g}^{-1}\right)$.

O teor de compostos fenólicos totais foi determinado utilizando o reagente Folin-Ciocalteau, segundo metodologia descrita por Singleton \& Rossi (1965). A absorbância foi medida a $765 \mathrm{~nm}$ em espectrofotômetro e os resultados expressos em miligramas de ácido gálico equivalente por 100 gramas em relação à massa fresca (mg AGE $\left.100 \mathrm{~g}^{-1}\right)$, utilizando curva padrão de ácido gálico, expressa pela Equação y $=0,0099 \mathrm{x}+0,0117\left(\mathrm{R}^{2}=0,9916\right)$.

A determinação da capacidade antioxidante foi realizada utilizando o radical ABTS (2,2'azino-bis-3etilbenzotiazolin 6-ácido sulfônico), de acordo com Re et al. (1999). A absorbância foi medida a $734 \mathrm{~nm}$ em espectrofotômetro e os resultados expressos em $\mu$ mol Trolox equivalente por grama ( $\mu \mathrm{mol} \mathrm{TE/g),} \mathrm{utilizando}$ curva padrão de Trolox, expressa pela Equação $y=-0,006 x+0,5799\left(R^{2}=0,9999\right)$.

\subsection{Análise estatística}

O delineamento experimental seguiu esquema fatorial $(2 \times 5)$ com dois tratamentos (pasteurização ou não) e cinco tempos de armazenamento a $-18{ }^{\circ} \mathrm{C}(0,30,60,90$ e 120 dias $)$, com três repetições. Os dados foram submetidos à análise de variância (ANOVA), seguida da aplicação do Teste de Tukey para comparação de médias, no nível de 5\% de probabilidade, no programa ASSISTAT 7.7 (Silva \& Azevedo, 2016). A análise de correlação simples (teste de correlação de Pearson +1/-1) foi calculada entre os teores de compostos fenólicos, carotenoides, vitamina $\mathrm{C}$ e capacidade antioxidante para as polpas de physalis pasteurizadas e não pasteurizadas, utilizando o teste $t$, no nível de $1 \%$ de probabilidade.

\section{Resultados e discussão}

$\mathrm{Na}$ Tabela 1, encontram-se expressos os resultados médios para as variáveis físico-químicas e químicas analisadas na polpa de physalis não pasteurizada e pasteurizada, armazenada sob congelamento.

Os resultados de $\mathrm{pH}$ para a polpa não pasteurizada e pasteurizada variaram durante o armazenamento. No entanto, ao se comparar o tempo zero e após 120 dias de armazenamento, os valores não diferiram estatisticamente $(\mathrm{p} \geq 0,05)$, apresentando, ao final do período, valores de $\mathrm{pH}$ de 3,98 $\pm 0,005$ e 4,03 $\pm 0,04$ na polpa não pasteurizada e pasteurizada, respectivamente. 
Tabela 1. Valores médios das variáveis físico-químicas e químicas das polpas de physalis não pasteurizadas e pasteurizadas, congeladas $\left(-18^{\circ} \mathrm{C}\right)$ por 120 dias.

\begin{tabular}{|c|c|c|c|c|c|c|}
\hline \multirow{2}{*}{ Variáveis } & \multirow{2}{*}{ Tratamentos } & \multicolumn{5}{|c|}{ Tempo de armazenamento (dias) } \\
\hline & & $\mathbf{0}$ & 30 & 60 & 90 & 120 \\
\hline \multirow{2}{*}{$\mathbf{p H}$} & Não Pasteurizada & $3,98 \pm 0,005^{\mathrm{cB}}$ & $4,03 \pm 0,005^{\mathrm{bA}}$ & $3,76 \pm 0,01 \mathrm{~dB}$ & $4,09 \pm 0,01$ aA & $3,98 \pm 0,005^{\mathrm{cA}}$ \\
\hline & Pasteurizada & $4,01 \pm 0,01 \mathrm{aA}$ & $4,03 \pm 0,01$ aA & $3,81 \pm 0,005^{\mathrm{bA}}$ & $4,06 \pm 0,087$ aA & $4,03 \pm 0,04$ aA \\
\hline \multirow{2}{*}{$\begin{array}{l}\text { Acidez Total Titulável } \\
\text { (g ácido cítrico/ } 100 \text { g) }\end{array}$} & Não Pasteurizada & $1,33 \pm 0,01 \mathrm{aB}$ & $1,35 \pm 0,05^{\mathrm{aA}}$ & $1,33 \pm 0,02^{\mathrm{aA}}$ & $1,24 \pm 0,04^{\mathrm{aA}}$ & $1,28 \pm 0,04^{\mathrm{aA}}$ \\
\hline & & $1,40 \pm 0,009$ aA & $1,35 \pm 0,06^{\mathrm{abA}}$ & $1,34 \pm 0,01 \mathrm{abA}$ & $1,30 \pm 0,01 \mathrm{bA}$ & $1,34 \pm 0,02^{\mathrm{abA}}$ \\
\hline \multirow{2}{*}{$\begin{array}{c}\text { Sólidos Solúveis Totais } \\
\left({ }^{\circ} \mathrm{Brix}\right)\end{array}$} & Não Pasteurizada & $14,30 \pm 0,17^{\mathrm{aB}}$ & $11,90 \pm 1,17^{\mathrm{bB}}$ & $12,86 \pm 0,41 \mathrm{abB}$ & $13,75 \pm 0,68$ aA & $13,53 \pm 0,11^{a b B}$ \\
\hline & Pasteurizada & $15,60 \pm 0,17^{\mathrm{aA}}$ & $14,13 \pm 0,25$ abA & $14,53 \pm 0,25$ abA & $13,24 \pm 1,00^{\mathrm{bA}}$ & $14,63 \pm 0,66^{\mathrm{abA}}$ \\
\hline \multirow{2}{*}{ Ratio SST/ATT } & Não Pasteurizada & $10,68 \pm 0,07$ abB & $8,78 \pm 1,20^{\mathrm{bA}}$ & $9,26 \pm 0,25$ abB & $11,07 \pm 0,94^{\mathrm{aA}}$ & $10,56 \pm 0,37 \mathrm{abA}$ \\
\hline & Pasteu & $11,08 \pm 0,19^{\mathrm{aA}}$ & $10,40 \pm 0,30 \mathrm{abA}$ & $10,78 \pm 0,14$ abA & $9,73 \pm 0,76^{\mathrm{bB}}$ & $10,84 \pm 0,67 \mathrm{abA}$ \\
\hline \multirow{2}{*}{ Umidade (\%) } & Não Pasteurizada & $80,32 \pm 0,38^{\mathrm{bA}}$ & $81,57 \pm 0,54^{a b A}$ & $82,76 \pm 0,94^{\mathrm{abA}}$ & $83,92 \pm 1,82^{\mathrm{aA}}$ & $84,01 \pm 0,44^{\mathrm{aA}}$ \\
\hline & Pasteurizada & $82,09 \pm 1,66^{\mathrm{aA}}$ & $82,82 \pm 2,35 \mathrm{aA}$ & $81,26 \pm 0,20^{\mathrm{aA}}$ & $81,56 \pm 0,75^{\mathrm{aA}}$ & $84,22 \pm 2,03$ aA \\
\hline \multirow{2}{*}{ Cinzas (\%) } & Não Pasteurizada & $1,53 \pm 0,18^{\mathrm{aA}}$ & $1,14 \pm 0,09^{\mathrm{bA}}$ & $1,26 \pm 0,03 \mathrm{abA}$ & $1,19 \pm 0,11 \mathrm{abA}$ & $1,14 \pm 0,15^{\mathrm{bA}}$ \\
\hline & Pasteurizada & $1,28 \pm 0,08^{\mathrm{aA}}$ & $0,96 \pm 0,06^{\mathrm{bA}}$ & $1,24 \pm 0,22^{a b A}$ & $1,15 \pm 0,15^{a b A}$ & $1,21 \pm 0,15$ abA \\
\hline \multirow{2}{*}{$\begin{array}{c}\text { Glicídios Redutores } \\
\text { (\% glicose) }\end{array}$} & Não Pasteurizada & $6,92 \pm 0,18^{\mathrm{bA}}$ & $12,49 \pm 0,59^{\mathrm{aA}}$ & $5,65 \pm 0,59^{\mathrm{cA}}$ & $5,86 \pm 0,32^{\mathrm{bcA}}$ & $6,04 \pm 0,44^{\mathrm{bcB}}$ \\
\hline & Pasteurizada & $6,10 \pm 0,25^{\mathrm{bB}}$ & $10,98 \pm 0,95^{\mathrm{aA}}$ & $4,67 \pm 0,23^{\mathrm{cA}}$ & $6,32 \pm 0,43{ }^{\mathrm{bA}}$ & $6,83 \pm 0,10^{\mathrm{bA}}$ \\
\hline
\end{tabular}

Média de três repetições \pm desvio padrão. Médias com letras minúsculas diferentes na linha diferem significativamente, em nível de $5 \%$, pelo Teste de Tukey. Médias com letras maiúsculas diferentes na coluna diferem significativamente, em nível de 5\%, pelo Teste de Tukey.

Os teores de ATT nas polpas não pasteurizadas e pasteurizadas de physalis não apresentaram variações significativas durante armazenamento, com valores de 1,28 $\pm 0,04$ e de 1,34 $\pm 0,02$, respectivamente, ao final do armazenamento. Esses teores foram próximos ao verificado por Oliveira (2016), ao avaliar frutos de physalis, de $1,25 \% \pm 0,03 \%$ de ácido cítrico.

Os resultados de SST nas polpas não pasteurizadas e pasteurizadas de physalis não apresentaram variações significativas durante armazenamento. Contudo, verificou-se diferença significativa $(p \leq 0,05)$ entre os tratamentos, sendo que a polpa pasteurizada apresentou maiores valores de ${ }^{\circ} \mathrm{Brix}$, indicando que o tratamento térmico promoveu a concentração do teor de SST (Amaro et al., 2002; Zillo et al., 2014). Rodrigues et al. (2014) encontraram em frutos de physalis valores de 13,81 $\pm 0,05^{\circ}$ Brix e Repo de Carrasco \& Zelada (2008) encontraram valores de 13,4 $\pm 0,2^{\circ}$ Brix, sendo próximos aos valores verificados nas polpas de physalis.

Os resultados encontrados para $\mathrm{pH}$, ATT e SST estão próximos aos verificados na literatura, podendo-se indicar que as polpas de physalis estão de acordo com a legislação vigente, que estabelece que as características físico-químicas deverão ser as provenientes do fruto de sua origem (Brasil, 2000).

A relação SST/ATT é uma das formas mais utilizadas para avaliação do sabor, sendo mais representativa que a medição isolada de açúcares ou da acidez (Chitarra \& Chitarra, 2005). Ao avaliar as polpas de physalis não pasteurizadas e pasteurizadas, observou-se que, embora tenham ocorrido variações na relação de SST/ATT ao longo dos dias de armazenamento, não foi observada diferença significativa $(p \geq 0,05)$ entre o primeiro e o último dia de armazenamento. Também não se verificou o efeito da pasteurização nessa relação. De acordo com Lima et al. (2009), o fruto de physalis deve apresentar a relação de SST/ATT $\geq 6,0$, a qual corresponde ao índice de maturação mínimo para comercialização. No entanto, essa variável deve estar associada a outras variáveis, como o conteúdo de sólidos solúveis, que deve ser de, no mínimo, $14{ }^{\circ}$ Brix. Desse modo, os resultados indicam que as polpas estão de acordo com o estabelecido na literatura, apresentando um equilíbrio entre os dois componentes.

Porém, quanto à estabilidade dos teores de umidade e cinzas durante o armazenamento, a polpa não pasteurizada apresentou aumento significativo $(\mathrm{p} \leq 0,05)$ da umidade, passando de $80,32 \% \pm 0,38 \%$ a valores de $84,01 \% \pm 0,44 \%$, e diminuição significativa $(\mathrm{p} \leq 0,05)$ no teor de cinzas, passando de $1,53 \pm 0,18$ para $1,14 \pm 0,15$. Não se verificou o efeito da pasteurização nos teores de umidade e cinzas $(p \geq 0,05)$ entre as polpas de physalis, ao longo dos dias de armazenamento. Oliveira (2016) encontrou valores médios de umidade de $83,02 \% \pm 0,94 \%$ em frutos de physalis e Repo de Carrasco \& Zelada (2008) verificaram uma 
umidade de 79,8\%, ambos os valores próximos ao das polpas do presente estudo. Para cinzas, Ramadan \& Mörsel (2004) e Repo de Carrasco \& Zelada (2008) encontraram valores de 1\%.

Os teores de açúcares redutores diferiram entre as polpas não pasteurizadas e pasteurizadas no tempo zero e após 120 dias de armazenamento. Notou-se aumento nos açúcares redutores após 30 dias de armazenamento em ambas as polpas analisadas, embora não tenha sido verificada diferença significativa $(p \geq 0,05)$ entre o tempo zero e 120 dias , com valores de 6,92 $\pm 0,18$ e 6,04 $\pm 0,44$ na polpa não pasteurizada e de 6,10 $\pm 0,25$ e 6,83 $\pm 0,10$ na polpa pasteurizada, respectivamente. $\mathrm{O}$ aumento dos valores de açúcares redutores aos 30 dias de armazenamento pode ter ocorrido devido à hidrólise da sacarose em meio ácido (Yuyama et al., 2008) ou também pode ser atribuído a erro experimental, pela dificuldade de identificar o ponto de viragem na titulação.Variações no teor de açúcares redutores entre polpas de frutas em função do período de armazenamento também foram encontradas por Evangelista \& Vieites (2006), ao avaliarem polpa de goiaba congelada, produzida por cinco empresas diferentes.

Na Figura 1, encontram-se os resultados para as variáveis de coloração avaliadas nas polpas de physalis não pasteurizadas e pasteurizadas, ao longo de 120 dias de armazenamento sob congelamento. Não se verificou diferença estatística $(p \geq 0,05)$ na luminosidade das polpas processadas. Comportamento semelhante também foi observado por Zillo et al. (2014) em polpa de uvaia. No entanto, a polpa não pasteurizada apresentou variações no valor de $L^{*}$ ao longo do armazenamento, porém sem apresentar diferença significativa entre o primeiro e último dia de armazenamento.
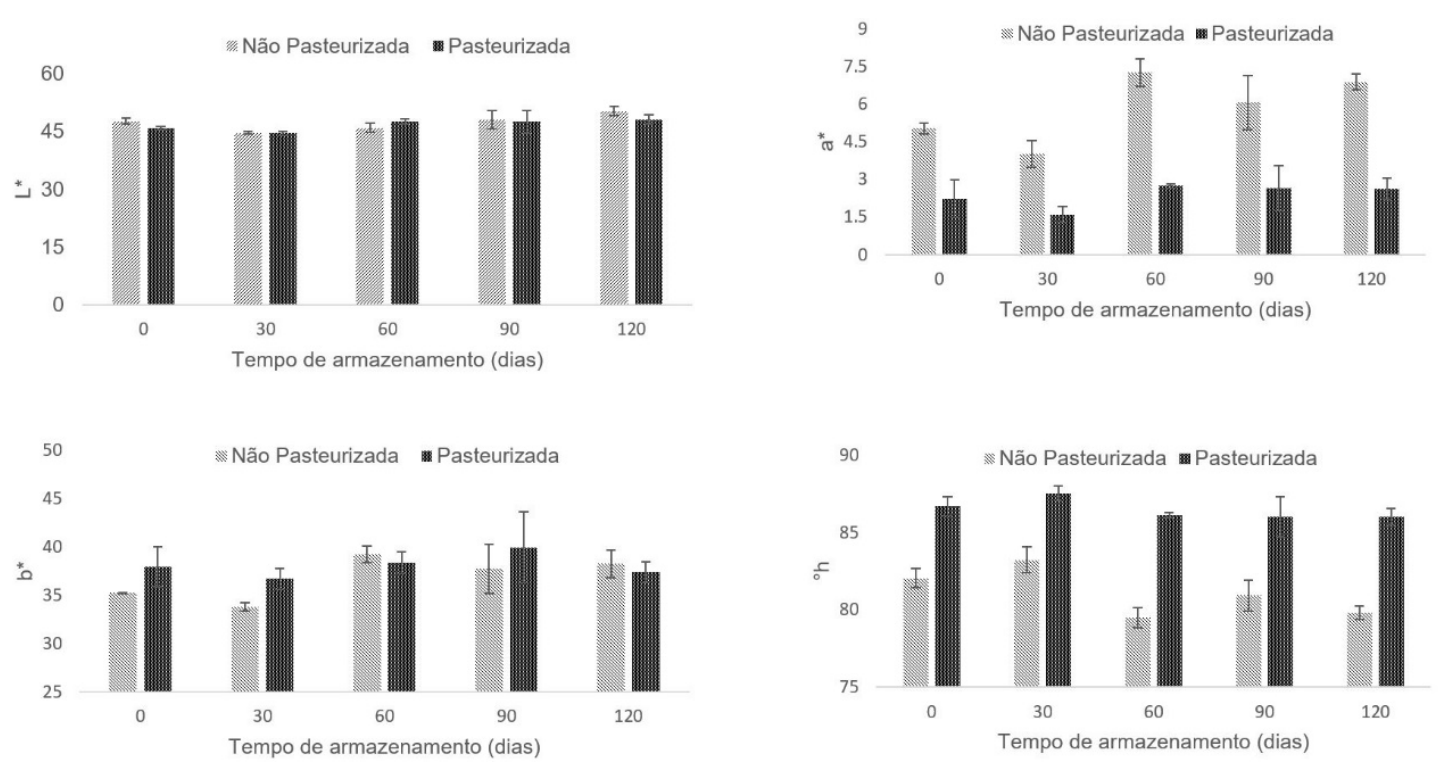

Figura 1. Médias dos parâmetros $\mathrm{L}^{*}, \mathrm{a}^{*}, \mathrm{~b}^{*} \mathrm{e}^{\circ} \mathrm{h}$ das polpas de physalis não pasteurizadas e pasteurizadas, congeladas $\left(-18^{\circ} \mathrm{C}\right)$ por 120 dias.

Verificou-se no parâmetro $\mathrm{a}^{*}$ da polpa não pasteurizada um aumento significativo $(\mathrm{p} \leq 0,05)$ durante o armazenamento, passando de 5,03 $\pm 0,22$, logo após o processamento, para 6,87 $\pm 0,31$, ao final de 120 dias. Na polpa pasteurizada esse parâmetro ficou estável, contudo, apresentou valores significativamente menores $(\mathrm{p} \leq 0,05)$ em todos os dias de armazenamento, indicando que a pasteurização provocou a perda de pigmentos instáveis responsáveis pela cor vermelha $\left(+\mathrm{a}^{*}\right)$.

A polpa pasteurizada não sofreu variações quanto ao parâmetro de cor $b^{*}$ ao longo dos dias de armazenamento, enquanto que a polpa que não sofreu o tratamento térmico apresentou um aumento desse parâmetro. No entanto, ao final dos dias de armazenamento, não se verificou diferença significativa $(p \geq 0,05)$ entre as polpas de physalis. Cabe ressaltar que a coordenada $\mathrm{a}^{*}$ das polpas de physalis apresentou valores médios menores que a coordenada $b^{*}$, o que indica a maior presença de pigmentação amarela. 
Foi observado que as polpas de physalis apresentam valores médios de ${ }^{\circ} \mathrm{h}$, que define o ângulo da tonalidade, entre os eixos $+\mathrm{a}^{*}$ (vermelho) e $+\mathrm{b}^{*}$ (amarelo). O ângulo de tonalidade inicia-se no eixo $+\mathrm{a}^{*}$ e é dado em graus, no qual zero seria $+\mathrm{a}^{*}$ (vermelho), 90 seria $+\mathrm{b}^{*}$ (amarelo), 180 seria $-\mathrm{a}^{*}$ (verde) e 270 seria -b* (azul) (Konica Minolta Sensing Inc., 1998). O valor do ${ }^{\circ} \mathrm{h}$ manteve-se constante nas polpas de physalis ao longo dos dias de armazenamento, com valores variando de $82,04 \pm 0,62$ a 79,80 $\pm 0,44$ na polpa não pasteurizada e de $86,69 \pm 0,62$ a $85,99 \pm 0,55$ na polpa pasteurizada, ou seja, o tempo de armazenamento não afetou esse parâmetro. No entanto, analisando-se os resultados, observou-se o efeito significativo da pasteurização $(\mathrm{p} \leq 0,05)$, que pode ser diretamente relacionado com os valores de $\mathrm{a}^{*}$, que também foram significativamente inferiores na polpa pasteurizada, apresentando valores de ${ }^{\mathrm{h}} \mathrm{h}$ mais próximos do amarelo.

Na Tabela 2, estão apresentados os resultados médios dos teores de carotenoides, vitamina C, compostos fenólicos e capacidade antioxidante das polpas de physalis não pasteurizadas e pasteurizadas, ao longo de 120 dias de armazenamento sob congelamento.

Tabela 2. Teor de carotenoides totais, vitamina $\mathrm{C}$, compostos fenólicos totais e capacidade antioxidante das polpas de physalis não pasteurizadas e pasteurizadas, congeladas $\left(-18^{\circ} \mathrm{C}\right)$ por 120 dias.

\begin{tabular}{|c|c|c|c|c|c|c|}
\hline \multirow{2}{*}{ Variáveis } & \multirow{2}{*}{ Tratamentos } & \multicolumn{5}{|c|}{ Tempo de armazenamento (dias) } \\
\hline & & $\mathbf{0}$ & 30 & 60 & 90 & 120 \\
\hline \multirow{2}{*}{ Vitamina C (mg/100 g) } & $\begin{array}{c}\text { Não } \\
\text { Pasteurizada }\end{array}$ & $\underset{\mathrm{aA}}{21,12 \pm 0,41}$ & $\begin{array}{c}3,04 \pm 0,44 \\
\text { bA }\end{array}$ & $2,80 \pm 0,97 \mathrm{bA}$ & $3,96 \pm 0,87^{b A}$ & $\underset{\mathrm{bA}}{3,72 \pm 0,41}$ \\
\hline & Pasteurizada & $\underset{\text { aA }}{26,16 \pm 4,00}$ & $\underset{b A}{2,23} \pm 1,93$ & $3,80 \pm 1,18^{b A}$ & $4,43 \pm 0,83^{\mathrm{bA}}$ & $\underset{\mathrm{bA}}{2,91 \pm 1,02}$ \\
\hline \multirow{2}{*}{ Carotenóides $(\mu \mathrm{g} / \mathrm{g})$} & $\begin{array}{c}\text { Não } \\
\text { Pasteurizada }\end{array}$ & $\underset{\mathrm{abA}}{5,28 \pm 1,06}$ & $\begin{array}{c}6,99 \pm 0,02 \\
\mathrm{aB}\end{array}$ & $\begin{array}{c}3,80 \pm 1,19 \\
\text { bcA }\end{array}$ & $1,17 \pm 0,51 \mathrm{dA}$ & $\underset{\mathrm{cdA}}{1,78 \pm 0,11}$ \\
\hline & Pasteurizada & $\begin{array}{c}6,51 \pm 1,06 \\
\mathrm{bA}\end{array}$ & $\underset{\mathrm{aA}}{10,44 \pm 0,82}$ & $\underset{\text { bcA }}{4,61 \pm 1,45}$ & $1,66 \pm 0,96^{\mathrm{cA}}$ & $\begin{array}{c}2,18 \pm 0,58 \\
\mathrm{cA}\end{array}$ \\
\hline \multirow{2}{*}{$\begin{array}{c}\text { Compostos fenólicos (mg } \\
\text { AGE/100 g) }\end{array}$} & $\begin{array}{c}\text { Não } \\
\text { Pasteurizada }\end{array}$ & $\begin{array}{c}60,87 \pm 2,63 \\
\text { aA }\end{array}$ & $\begin{array}{c}28,99 \pm 4,54 \\
\text { cB }\end{array}$ & $\underset{\text { abA }}{54,80 \pm 1,34}$ & $\underset{\mathrm{abA}}{59,45 \pm 9,13}$ & $\begin{array}{c}47,32 \pm 0,64 \\
\mathrm{bA}\end{array}$ \\
\hline & Pasteurizada & $\underset{\mathrm{aA}}{64,35 \pm 3,39}$ & $\begin{array}{c}42,02 \pm 8,56 \\
\mathrm{cA}\end{array}$ & $\begin{array}{c}59,79 \pm 4,76 \\
\text { abA }\end{array}$ & $\underset{\text { bcA }}{51,43 \pm 0,35}$ & $\begin{array}{c}44,20 \pm 1,37 \\
\mathrm{cB}\end{array}$ \\
\hline \multirow{2}{*}{$\begin{array}{c}\text { Capacidade Antioxidante ( } \mu \mathrm{mol} \\
\mathrm{TE} / \mathrm{g})\end{array}$} & $\begin{array}{c}\text { Não } \\
\text { Pasteurizada }\end{array}$ & $\underset{\mathrm{bA}}{1,98 \pm 0,22}$ & $\begin{array}{c}6,92 \pm 0,57 \\
\mathrm{aA}\end{array}$ & $1,95 \pm 0,53 \mathrm{bA}$ & $0,01 \pm 0,01^{\mathrm{cA}}$ & $\begin{array}{l}0,00 \pm 0,00 \\
\mathrm{cA}\end{array}$ \\
\hline & Pasteurizada & $\underset{b A}{2,44} \pm 0,19$ & $\begin{array}{c}7,50 \pm \underset{\mathrm{aA}}{0,52} \\
0,52\end{array}$ & $1,39 \pm 0,24^{\mathrm{cA}}$ & $0,06 \pm 0,06 \mathrm{dA}$ & $\underset{\mathrm{dA}}{0,00 \pm 0,02}$ \\
\hline
\end{tabular}

Média de três repetições \pm desvio padrão. Médias com letras minúsculas diferentes na linha diferem significativamente, em nível de $5 \%$, pelo Teste de Tukey. Médias com letras maiúsculas diferentes na coluna diferem significativamente, em nível de 5\%, pelo Teste de Tukey.

Houve redução significativa $(\mathrm{p} \leq 0,05)$ no teor de carotenoides ao longo do armazenamento para as polpas não pasteurizadas e pasteurizadas. $O$ teor de carotenoides na polpa não pasteurizada passou de $5,28 \mu \mathrm{g} / \mathrm{g}$ para $1,78 \mu \mathrm{g} / \mathrm{g}$ e, na polpa pasteurizada, de $6,51 \mu \mathrm{g} / \mathrm{g}$ para $2,18 \mu \mathrm{g} / \mathrm{g}$, após os 120 dias de armazenamento. Assim, as perdas ao longo do armazenamento representam uma diminuição de $66,29 \%$ e $66,51 \%$ do conteúdo inicial de carotenoides nas polpas não pasteurizadas e pasteurizadas, respectivamente.

As perdas dos carotenoides se devem à oxidação enzimática e não enzimática. A perda por oxidação enzimática ocorre logo após a ruptura das estruturas celulares, enquanto que a oxidação não enzimática normalmente se caracteriza por uma fase lag, seguida de um decréscimo dos carotenoides (Rodriguez-Amaya et al., 2008). Deste modo, em ambas as polpas, verificou-se que a principal causa do decréscimo considerável dos carotenoides foi a oxidação não enzimática.

Observou-se, apenas aos 30 dias de armazenamento, um efeito significativo $(\mathrm{p} \leq 0,05)$ do tratamento térmico, sendo que a polpa pasteurizada apresentou um maior teor de carotenoides. Maia et al. (2007), ao estudarem o efeito do processamento sobre componentes do suco de acerola, observaram um aumento no teor de carotenoides no suco pasteurizado e indicaram que esse fato pode ser atribuído ao rompimento da estrutura da parede celular. Silva et al. (2015) observaram que, durante o armazenamento de polpas de 
araticum, os teores de carotenoides reduziram até o $30^{\circ}$ dia de armazenamento. Após esse período, verificouse aumento no teor de carotenoides, sugerindo que as alterações no teor de carotenoides durante o armazenamento sob congelamento decorrem de processos complexos, os quais são influenciados por diversos fatores, tais como matriz alimentar, composição química, processamento térmico, os quais afetam a concentração desses compostos de forma inconsistente. Hoffmann et al. (2017) também verificaram variações nos teores de carotenoides ao longo de 12 meses de armazenamento de polpa de butiá.

Houve redução significativa $(\mathrm{p} \leq 0,05)$ no teor de vitamina $\mathrm{C}$ ao longo do período de armazenamento para ambas as polpas. Os valores passaram de $21,12 \mathrm{mg} / 100 \mathrm{~g}$ para $3,72 \mathrm{mg} / 100 \mathrm{~g}$ na polpa não pasteurizada e de $26,12 \mathrm{mg} / 100 \mathrm{~g}$ para $2,91 \mathrm{mg} / 100 \mathrm{~g}$ na polpa pasteurizada. As perdas ao longo do armazenamento a $-18{ }^{\circ} \mathrm{C}$ representam uma diminuição de $82,38 \%$ e $88,88 \%$ do conteúdo inicial de vitamina $\mathrm{C}$ nas polpas não pasteurizadas e pasteurizadas, respectivamente. Não foi verificado efeito significativo $(p \geq 0,05)$ do tratamento de pasteurização.

Monteiro et al. (2005), ao estudarem polpa de maracujá processada e armazenada sob congelamento, encontraram uma redução no teor de vitamina $\mathrm{C}$ entre $77,8 \%$ a $82,3 \%$ em polpas pasteurizadas em diferentes temperaturas, ao fim do período de estocagem, valores esses próximos dos encontrados neste estudo. Resultado bastante semelhante também foi encontrado por Silva et al. (2013), que verificaram teores de vitamina $\mathrm{C}$ de $25,55 \mathrm{mg} / 100 \mathrm{~g}$ em frutos de physalis no momento da colheita e, após 28 dias de armazenamento a $5{ }^{\circ} \mathrm{C}$, houve redução nos teores de vitamina $\mathrm{C}$, chegando ao valor médio de $6,66 \mathrm{mg} / 100$ g. A diminuição do teor de vitamina $\mathrm{C}$ ao longo do armazenamento pode ser explicada pelo fato de a vitamina C ser uma vitamina hidrossolúvel e de fácil oxidação, principalmente pela aplicação do calor ou armazenamento a baixas temperaturas (Sucupira et al., 2012).

O teor de compostos fenólicos sofreu redução significativa $(\mathrm{p} \leq 0,05)$ ao longo do armazenamento sob congelamento, independentemente da aplicação ou não do tratamento térmico. Os valores passaram de $60,87 \pm 2,63 \mathrm{mg} \mathrm{AGE} / 100 \mathrm{~g}$ para 47,32 $\pm 0,64 \mathrm{mg}$ AGE/100 $\mathrm{g}$ na polpa não pasteurizada e de $64,35 \pm 3,39 \mathrm{mg}$ AGE/100 g para 44,20 $\pm 1,37 \mathrm{mg} \mathrm{AGE/100} \mathrm{g} \mathrm{na} \mathrm{polpa} \mathrm{pasteurizada.} \mathrm{As} \mathrm{perdas,} \mathrm{ao} \mathrm{longo} \mathrm{do} \mathrm{armazenamento,}$ representam uma diminuição de $22,26 \%$ e $31,31 \%$ do teor inicial de compostos fenólicos nas polpas não pasteurizadas e pasteurizadas, respectivamente. Lima (2010), em estudo com polpa de acerola orgânica pasteurizada e congelada, encontrou perda de $68,1 \%$ no teor de compostos fenólicos totais durante o armazenamento, evidenciando que o processamento e o armazenamento sob congelamento interferem na estabilidade desses compostos.

Após 120 dias de armazenamento ocorreu diferença significativa $(\mathrm{p} \leq 0,05)$ entre os tratamentos, indicando que a pasteurização promoveu a perda de compostos fenólicos. O menor teor de compostos fenólicos encontrado pode ser associado aos menores valores de a* verificados na polpa pasteurizada.

Com relação à capacidade antioxidante, , verificou-se um decréscimo significativo $(p \leq 0,05)$ nos teores, ao longo do armazenamento, tanto para a polpa não pasteurizada como para a polpa pasteurizada. Ao final do armazenamento, os valores da capacidade antioxidante não foram quantificados. Não se verificou efeito significativo do tratamento térmico.

A ocorrência de coeficientes de correlação de Pearson positivos, muito fortes, entre os carotenoides e a capacidade antioxidante, variando de 0,89 e 0,92 , nas polpas não pasteurizadas e pasteurizadas, respectivamente, indica que os carotenoides representam estatisticamente os componentes responsáveis pela capacidade antioxidante da polpa de physalis congelada. Embora tenha ocorrido a diminuição da vitamina $\mathrm{C}$, a correlação existente entre o ácido ascórbico e a capacidade antioxidante é desprezível, com valores de $-0,07$ e $-0,03$, para as polpas não pasteurizadas e pasteurizadas, respectivamente.

Com relação aos compostos fenólicos, a polpa não pasteurizada apresentou correlação de Pearson negativa, moderada e significativa $(\mathrm{p} \leq 0,01)$ de $-0,75$, e a polpa pasteurizada apresentou correlação de Pearson negativa, fraca e significativa de $-0,31$, ou seja, os teores de compostos fenólicos mantiveram-se altos, enquanto que a capacidade antioxidante diminuiu. 


\section{Conclusões}

Com relação às características físico-químicas e químicas das polpas de physalis mantidas a $-18{ }^{\circ} \mathrm{C}$, observou-se que o armazenamento sob congelamento não ocasionou perdas significativas para as variáveis de $\mathrm{pH}$, ATT, SST e glicídeos redutores e que a aplicação do tratamento térmico promoveu variações significativas para os SST e glicídios redutores ao final do período de armazenamento, observando-se maiores teores. Analisando-se os resultados dos parâmetros de cor, foram observadas diferenças significativas $(\mathrm{p} \leq 0,05)$ entre as polpas de physalis não pasteurizadas e pasteurizadas para os parâmetros $\mathrm{a}^{*} \mathrm{e}^{\circ} \mathrm{h}$, indicando a perda de pigmentos vermelhos.

Quanto à estabilidade dos compostos antioxidantes, verificou-se redução nos teores de carotenoides, vitamina $\mathrm{C}$ e compostos fenólicos totais, ao longo dos 120 dias de armazenamento a $-18^{\circ} \mathrm{C}$ nas polpas de physalis não pasteurizadas e pasteurizadas. Além disso, houve redução significativa na capacidade antioxidante durante $\mathrm{o}$ armazenamento para ambas as polpas.

\section{Referências}

Amaro, A. P., Bonilha, P. R. M., \& Monteiro, M. (2002). Efeito do tratamento térmico nas características físico- químicas e microbiológicas da polpa de maracujá. Alimentos e Nutrição, 13, 151-162.

Bolzan, R. P., Cuquel, F. L., \& Lavoranti, O. J. (2011). Armazenamento refrigerado de Physalis. Revista Brasileira de Fruticultura (Vol. Especial), 577-583.

Brasil. (2000, janeiro 10). Dispõe sobre o regulamento técnico geral para fixação dos padrões de identidade e qualidade para polpa de fruta (e suco de fruta) (Instrução Normativa № 01, de 07 de janeiro de 2000). Diário Oficial [da] República Federativa do Brasil, Brasília.

Carvalho, A. V., Mattietto, R. A., \& Beckman, J. C. (2017). Estudo da estabilidade de polpas de frutas tropicais mistas congeladas utilizadas na formulação de bebidas. Brazlilian Journal of Food Technology, 20, e2016023. http://dx.doi.org/10.1590/1981-6723.2316.

Chitarra, M. I. F., \& Chitarra, A. B. (2005). Pós-colheita de frutas e hortaliças: fisiologia e manuseio (2. ed.). Lavras: FAEPE.

Evangelista, R. M., \& Vieites, R. L. (2006). Avaliação da qualidade de polpa de goiaba congelada comercializada na cidade de São Paulo. Segurança Alimentar e Nutricional, 13(2), 76-81. http://dx.doi.org/10.20396/san.v13i2.1834

Fellows, P. J. (2006). Tecnologia do processamento de alimentos: princípios e prática (2. ed.). Porto Alegre: Artmed.

Fischer, G., Herrera, A., \& Almanza, P. J. (2011). Cape gooseberry (Physalis peruviana L.) In E. M. Yahia (Ed.). Postharvest biology and technology of tropical and subtropical fruits (Vol. 2, pp. 374-396). Oxford: Woodhead Publishing. http://dx.doi.org/10.1533/9780857092762.374.

Hoffmann, J. F., Zandoná, G. P., Santos, P. S., Dallmann, C. M., Madruga, F. B., Rombaldi, C. V., \& Chaves, F. C. (2017). Stability of bioactive compounds in butiá (Butia odorata) fruit pulp and nectar. Food Chemistry, 237, 638-644. PMid:28764046. http://dx.doi.org/10.1016/j.foodchem.2017.05.154

Instituto Adolfo Lutz - IAL. (2008). Normas Analíticas do Instituto Adolfo Lutz (3. ed., Vol. 1). São Paulo: IMESP.

Konica Minolta Sensing Inc. (1998). Comunicação precisa de cor: controle de qualidade da percepção à instrumentação (59 p.). Osaka: AEBDPK®.

Lima, C. S. M., Severo, J., Andrade, S. B., Affonso, L. B., Rombaldi, C. V., \& Rufato, A. D. R. (2013). Qualidade pós-colheita de Physalis sob temperatura ambiente e refrigeração. Revista Ceres, 60(3), 311-317. http://dx.doi.org/10.1590/S0034737X2013000300002

Lima, C. S. M., Severo, J., Manica-Berto, R., Silva, J. A., Rufato, L., \& Rufato, A. R. (2009). Características físico-químicas de Physalis em diferentes colorações do cálice e sistemas de condução. Revista Brasileira de Fruticultura, 31(4), 1060-1068. http://dx.doi.org/10.1590/S0100-29452009000400020

Lima, R. M. T. (2010). Avaliação da estabilidade química, físico-química e microbiológica de polpas de acerola orgânica pasteurizada e não-pasteurizada (Dissertação de mestrado). Universidade Federal do Ceará, Fortaleza.

Lima, R. M. T., Figueiredo, R. W., Maia, G. A., Sousa, P. H. M., Figueiredo, E. A. T., \& Rodrigues, C. S. (2012). Estabilidade química, físico-química e microbiológica de polpas de acerola pasteurizadas e não-pasteurizadas de cultivo orgânico. Ciência Rural, 42(2), 367-373. http://dx.doi.org/10.1590/S0103-84782012005000005

Maia, G. A., Sousa, P. H. M., Santos, G. M., Silva, D. S., Fernandes, A. G., \& Prado, G. M. (2007). Efeito do processamento sobre componentes do suco de acerola. Revista Ciência e Tecnologia de Alimentos, 27(1), 130-134. http://dx.doi.org/10.1590/S0101-20612007000100023

Matta, V. M., Freire Junior, M., Cabral, L. M. C., \& Furtado, A. A. L. (2005). Polpa de fruta congelada. Brasília: Embrapa Informação Tecnológica. 
Monteiro, M., Amaro, A. P., \& Bonilha, P. R. M. (2005). Avaliação físico-química e microbiológica da polpa de maracujá processada e armazenada sob refrigeração. Alimentos e Nutrição, 16(1), 71-76.

Oliveira, S. F. (2016). Estudo das propriedades físico-químicas e avaliação de compostos bioativos em Physalis peruviana $L$. (Dissertação de mestrado). Instituto Politécnico de Viseu, Viseu.

Ramadan, M. F. (2011). Bioactive phytochemicals, nutritional value, and functional properties of capegooseberry (Physalis peruviana): an overview. Food Research International, 44(7), 1830-1836. http://dx.doi.org/10.1016/j.foodres.2010.12.042 Ramadan, M. F., \& Mörsel, J. T. (2004). Goldenberry: a novel fruit source of fat soluble bioactives. Inform, 15, 130-131.

Rawson, A., Patras, A., Tiwari, B. K., Noci, F., Koutchma, T., \& Brunton, N. (2011). Effect of thermal and non thermal processing technologies on the bioactive content of exotic fruits and their products: review of recent advances. Food Research International, 44(7), 1875-1887. http://dx.doi.org/10.1016/j.foodres.2011.02.053

Re, R., Pellegrini, N., Proteggente, A., Pannala, A., Yang, M., \& Rice-Evans, C. (1999). Antioxidant activity applying an improved ABTS radical cátion decolorization assay. Free Radical Biology \& Medicine, 26(9-10), 1231-1237. PMid:10381194. http://dx.doi.org/10.1016/S0891-5849(98)00315-3

Repo de Carrasco, R. R., \& Zelada, C. R. E. (2008). Determinación de la capacidad antioxidante y compuestos bioactivos de frutas nativas peruanas. Revista de la Sociedad Química del Perú, 74, 108-124.

Ribeiro, L. O., Pontes, S. M., Ribeiro, A. P. O., Pacheco, S., Freitas, S. P., \& Matta, V. M. (2017). Avaliação do armazenamento a frio sobre os compostos bioativos e as características físico-químicas e microbiológicas do suco de umbu pasteurizado. Brazilian Journal of Food Technology, 20, e2015095. http://dx.doi.org/10.1590/1981-6723.9515.

Rockenbach, I. I., Rodrigues, E., Cataneo, C., Gonzaga, L. V., Lima, A., Mancini-Filho, J., \& Fett, R. (2008). Ácidos fenólicos e atividade antioxidante em Fruto de physalis peruviana L. Alimentos e Nutrição, 19(3), 271-276.

Rodrigues, F. A., Penoni, E. S., Soares, J. D. R., Silva, R. A. L., \& Pasqual, M. (2014). Caracterização física, química e físicoquímica de physalis cultivada em casa de vegetação. Ciência Rural, 44(8), 1411-1414. http://dx.doi.org/10.1590/0103$8478 \mathrm{cr} 20130743$

Rodriguez-Amaya, D. (2001). A guide to carotenoid analysis in foods. Washington: International Life Sciences Institute. Rodriguez-Amaya, D., Kimura, M., \& Amaya-Farfan, J. (2008). Fontes brasileiras de carotenoides: Tabela brasileira de composição de carotenoides em alimentos. Brasília: MMA/SBF.

Silva, D. F., Villa, F., Barp, F. K., Rotili, M. C. C., \& Stumm, D. R. (2013). Conservação pós-colheita de fisális e desempenho produtivo em condições edafoclimáticas de Minas Gerais. Revista Ceres, 60(6), 826-832. http://dx.doi.org/10.1590/S0034737X2013000600011

Silva, F. A. S., \& Azevedo, C. A. V. (2016). The Assistat Software Version 7.7 and its use in the analysis of experimental data. African Journal of Agricultural Research, 11(39), 3733-3740. http://dx.doi.org/10.5897/AJAR2016.11522

Silva, L. L., Cardoso, L. M., \& Pinheiro-Sant'ana, H. M. (2015). Influênica do branqueamento, pasteurização e congelamento nas características físico-químicas, nos carotenoides e no valor de vitamina A de polpa de araticum (Annona crassiflora Mart.). Revista do Instituto Adolfo Lutz, 74(1), 30-38.

Singleton, V. L., \& Rossi, J. A. (1965). Colorimetry of total phenolics with osphomolybdic-phosphotungstic acid reagents. American Journal of Enology and Viticulture, 16, 144-158.

Sucupira, N. R., Xerez, A. C. P., \& Sousa, P. H. M. (2012). Perdas vitamínicas durante o tratamento térmico de alimentos. UNOPAR Científica. Ciências Biológicas e da Saúde, 14(2), 121-128.

Yuyama, L. K. O., Pantoja, L., Maeda, R. N., Aguiar, J. P. L., \& Silva, S. B. (2008). Desenvolvimento e aceitabilidade de geleia dietética de cubiu (Solanum sessiflorum Dunal). Food Science and Technology (Campinas), 28(4), 929-934. http://dx.doi.org/10.1590/S0101-20612008000400026

Zillo, R. R., Silva, P. P. M., Zanatta, S., \& Spoto, M. H. F. (2014). Parâmetros físico-químicos e sensoriais de polpa de uvaia (Eugenia Pyriformis) submetidas à pasteurização. Bioenergia em revista. Diálogos, 4(2), 20-33.

Financiamento: Nenhum. 